\title{
Evaluation of the Effect of Platelet-Rich Plasma on Post-Burn Scars
}

\author{
Malek Elsayed*, Mohamed Abdel Moaty, Amr Moghazy and Omar Salah Eldeen \\ Surgery Department, Suez Canal University Hospitals, Faculty of Medicine, Suez Canal University, Egypt
}

Submission: August 06, 2017; Published: August 16, 2017

*Corresponding author: Malek Ahmed Mohamed Elsayed, Department of Surgery, Plastic Surgery Unit, Suez Canal University Hospitals, Ismailia, Egypt, Tel: +201000919368; Email: dr.malek.ahmed@gmail.com

\section{Abstract}

Introduction: Management of post burn scars has a great challenge. Treatments have progressed from rudimentary methods such as excision and radiotherapy to less invasive techniques such as intralesional injection, pressure therapy and Silicone-based preparations.

Objectives: Our main objective was to evaluate the effect of platelet-rich plasma (PRP) in post-burn scars regarding the aesthetic and symptoms improvement as well as rate of complications. Also to compare the efficacy of intralesional PRP injection as compared to control group treated according the standard guidelines in the treatment of post burn scars.

Methods: There were 8 male patient (42\%) and 11 female patients (58\%) of the PRP group, and 6 male patients (31.6\%) and 13 female patients (68.4\%) of the Silicone group. Assessment of scar was done including history, clinical examination using Patient and Observer Scar Assessment Scale. Patients with group 1 treated with one session of PRP injection every month (a total of three sessions).Patients with group 2 treated with Silicone-based products application for at least three months. The follow-up took place after 1 week, 1, 3, 6 months.

Results: Treatment of patients with post burn mature scar by intralesional PRP injection was found to be better than treatment of the patients by Silicone-based products. Especially in itching, pigmentation and pliability, on the other hand treatment with Silicone-based products showed better results than treating with PRP in improving the thickness of the scar:

Conclusion: This novel treatment appeared to be safe and effective for scar treatment. Further studies are needed to explore the potential use of this treatment.

Keywords: Platelet-Rich Plasma; PRP; Burn; Scars

\section{Introduction}

The healing process comprehends four stages, namely: clotting, inflammation, cell proliferation, matrix repair and epithelialization, and finally remodeling of the cicatricial tissue [1]. Following the injury, platelets are stimulated to aggregate and to secrete growth factors, cytokines and other homeostatic factors required for clotting cascade which characterize, therefore, the first stage [2]. Clotting and platelet degranulation lead to the inflammatory stage by release of serotonin and histamine, bioactive factors which increase the capillary permeability and allow the arrival of larger inflammatory cells to the place of wound, such as leukocytes, macrophages and neutrophils, which act on the phagocytosis of the products resulting from the cellular lysis [3] When this stage is over, the number of anti-inflammatory cells is reduced and fibroblasts produce collagen, elastin and other components of the matrix [1].
Residual epithelial cells or those which have migrated to the injured area multiply themselves and form scars. The scar is then remodeled from a balance between the degradation and the production of extracellular matrix, besides the reduction of fibroblasts and capillaries by apoptosis process [1]. Growth factors have been known since the 1950s [4], however, in 1982 only growth factors deriving from platelets themselves were described in detailed form [5]. The first clinical use described in the literature dates back to 1987, when Ferrari et al. described the use of autologous PRP as an additional element for transfusion in cardiac surgeries, for the purpose of eliminating the use of homologous products. However, in the 90's decade PRP started to be widely used as an adjuvant in many surgical procedures, mainly in the areas of dentistry and orthopedics [6].

The rational for the clinical use of PRP is based on its ability to stimulate the production and, subsequently, increase the 
concentration of growth factors and secretion of proteins which are able to improve the healing process at the cellular level [2]. In the context of inflammatory lesions, PRP acts by secretion of growth factors, inflammatory mediators such as cytokines and chemokines, as well as expression of chemokine receptors. The enriched concentration of activated platelets presents a balance of pro- and anti-inflammatory factors well-orchestrated in order to resolve inflammation. In this sense, the PRP is capable of modulating secretion and recruitment of inflammatory cells, such as monocytes and leukocytes, in the injury site. Therefore, the therapeutic action and tissue regeneration PRPmediated process seems to result from the control of the local inflammatory process [7].

PRP has been used for about 20 years in different surgical fields of medicine to improve wound healing and repair. Platelet growth factors have shown its ability to improve healing in many clinical studies, particularly in periodontal surgery, orthopedics and trauma surgery, and plastic surgery [8]. Despite this richness in the field of stimulation of healing, there is lack of literature addressing the clinical experience and outcomes of scars treatment, during the maturation and remodeling phases, using PRP. Therefore, the purpose of this study is to evaluate the effect of PRP on scars through modulating the inflammatory state of this anomaly of scarring processes following burn.

\section{Methods}

\section{Subjects}

The current prospective randomized clinical controlled trial was conducted at the outpatient clinic of plastic surgery of Suez Canal University Hospitals, Faculty of Medicine, Suez Canal University in the period between June 2016 and February 2017 after the approval of the Research Ethical Committee (REC), Faculty of Medicine, Suez Canal University was obtained A written informed consent for treatment together with photography from all participants before enrollment in the study was obtained. A total of thirty-eight patients with post-burn scars fulfilling the inclusion criteria were included in the study. The selection of the patients in this study was done according to the following criteria:

a. Inclusion Criteria

i. Patients with mature post-burn scars.

ii. Patients with post-burn scars irrespective to sex, age and scar location.

iii. Scars ranged from $2 \mathrm{~cm}$ to $15 \mathrm{~cm}$ in diameter.

iv. Scar duration ranged from 6 months to 4 years.

v. Patients signed the informed consent form prior to participation.

b. Exclusion Criteria i. Patients known to have thrombocytopenia, platelet dysfunction syndrome, septicemia, hypofibrinogenemia, recent fever condition, anemia, cancer.

ii. Patients with skin lesions in the area of the injection.

iii. Current intake of oral anticoagulants, platelet aggregation inhibitors, abnormal bleeding history and use of corticosteroids (in up to 6weeks before the procedure), or non-steroid anti-inflammatory (48 hours before procedure).

The sample divided into two equal groups: Group 1 involved 19 patients who treated with PRP intralesional injection. Group 2 involved 19 patients who treated as a control group with Silicone-based products application. Randomization done using card technique in which every patient took a random number 1-38: Patients with odd number subjected to group 1. Patients with even numbers subjected to group 2 .

\section{Procedure}

Questionnaire designed to collect data about the personal data, preoperative, intraoperative and postoperative evaluation. All patients were interviewed and their personal data taken, and recorded. Those data included patient name, age, sex, phone number, chronic illnesses, blood diseases and skin diseases. After obtaining informed consent from the patients, a blood sample was aspirated and collected using a blood collection tubes from a peripheral vein which was equipped with a butterfly $21 \mathrm{G}$ needle; vacutainer kit (to avoid direct contact with blood samples). The blood sample tube contains sodium citrates as an anticoagulant. The tubes were centrifuged at $400 \times \mathrm{g}$ for $10 \mathrm{~min}$. After this stage, three layers clearly demarcated: plasma, red blood cells, and an intermediate zone.

The plasma is at the top with the platelets, the red blood cells are at the bottom because of their heavier density; and the fine, whitish intermediate zone consists of larger platelets and leukocytes and is called the buffy coat. Using an $18 \mathrm{G}$ needle, the upper portion of plasma with platelets drawn off, and the buffy coat placed into another tube, this time with no additives. The tubes were centrifuged again, this time at $800 \times \mathrm{g}$, for the same length of time ( $\mathrm{T}=10 \mathrm{~min}$ ). After this final centrifugation, the tubes contained the platelet sedimentation and some red blood cells (erythrocyte- platelet clump). The volume reduced at this stage by removing two-thirds of the total plasma volume.

Meanwhile, objective examinations carried out, photographs were taken and a personal folder filled in. the blood samples were centrifugally separated, thus obtaining a two-part plasma: the upper part, consisting of PPP; and the lower part, consisting of PRP. The PPP first gently aspirated, to avoid its mixing up with the PRP. The residual of PRP subsequently aspirated from the test tube and prepared for activation by calcium gluconate Figure 1 in the proportion of $0.1 \mathrm{~mL}$ per $0.9 \mathrm{~mL}$ of PRP. The PRP solution finally injected within next seven minutes (Figure 2). 

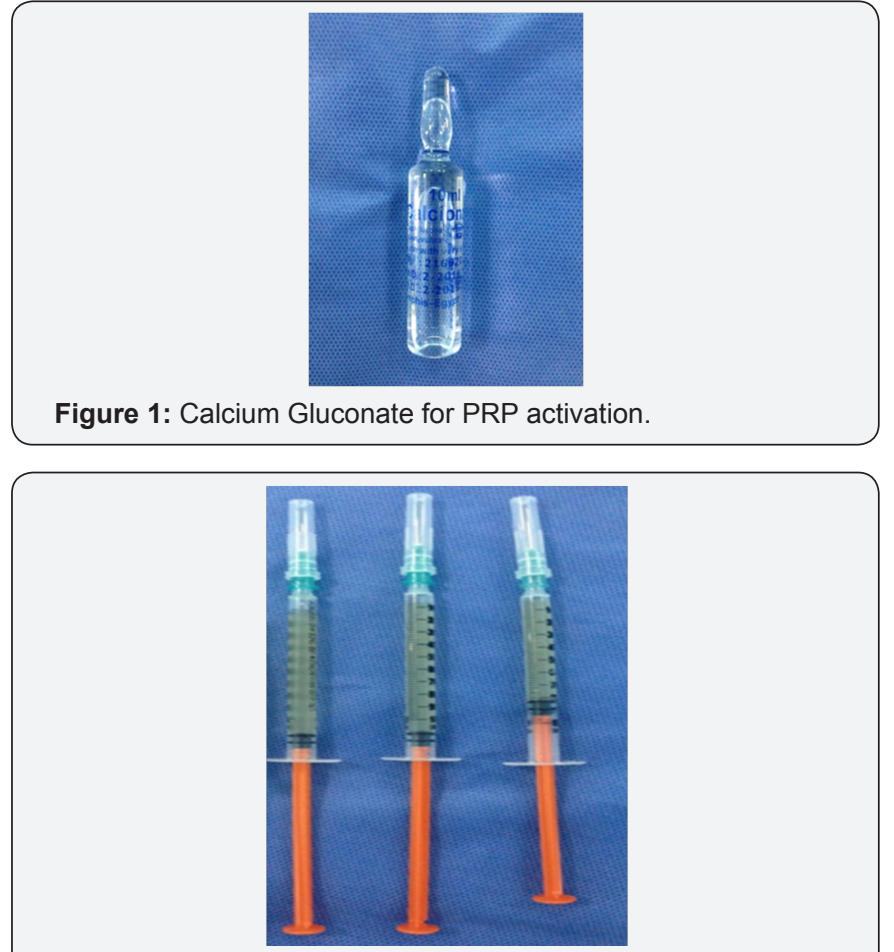

Figure 2: Prepared PRP for injection.

Patients with group 1 treated with one session of PRP injection every month (a total of three sessions). A $3 \mathrm{~mL}$ syringe with a $30 \mathrm{G}$ needle was used. The PRP solution finally injected within next seven minutes. The injection techniques, $0.2 / 0.3 \mathrm{~mL}$ solution administered using a "linear retrograde and fanning" technique with two or three retrograde injections, called "tunnelling": a back-and-forth movement of the needle which first creates a tunnel, a track and then fills it with the solution. The "tunnelling" technique takes advantage of the restoration of the mild trauma provoked.

Patients with group 2 treated with Silicone-based products application for at least three months. Silicone gel or sheeting applied over the scars and maintained for at least three months. For Silicone gel sheeting, a minimum 12-hour daily wear time advocated and, if possible, continuous 24-hour coverage with washing twice daily. Use of Silicone gel in cream or ointment form preferred to Silicone gel sheeting for high-mobility or large areas, use on the face, or in hot humid climates [9]. Treated areas gently massaged and molded with hydrating cream after each session. After the treatment the patients returned to normal activities the very next day. The patients were discharged at the same day; at departure they used emollient creams and creams with SPF 50 + for 1 month. The follow-up took place after 1 week, 1, 3, 6 months.

\section{Results}

Subsequently, the study included 38 participants with postburn scars. The sample was divided randomly into two groups each containing 19 participants, the first group was treated with PRP intralesional injection and the other group was treated as a control group with Silicone-based products application. There were 8 male patient (42\%) and 11 female patients (58\%) of the PRP group, and 6 male patients (31.6\%) and 13 female patients (68.4\%) of the Silicone group. Mean patients age of PRP group was 19.5 \pm 7.7 years (range 4-35). Mean patients age of Silicone group was $20 \pm 11.7$ years (range 3-37). Scalds were the most common cause of burns among both groups; $31.58 \%$ among the whole patient, flame burns were second in place.
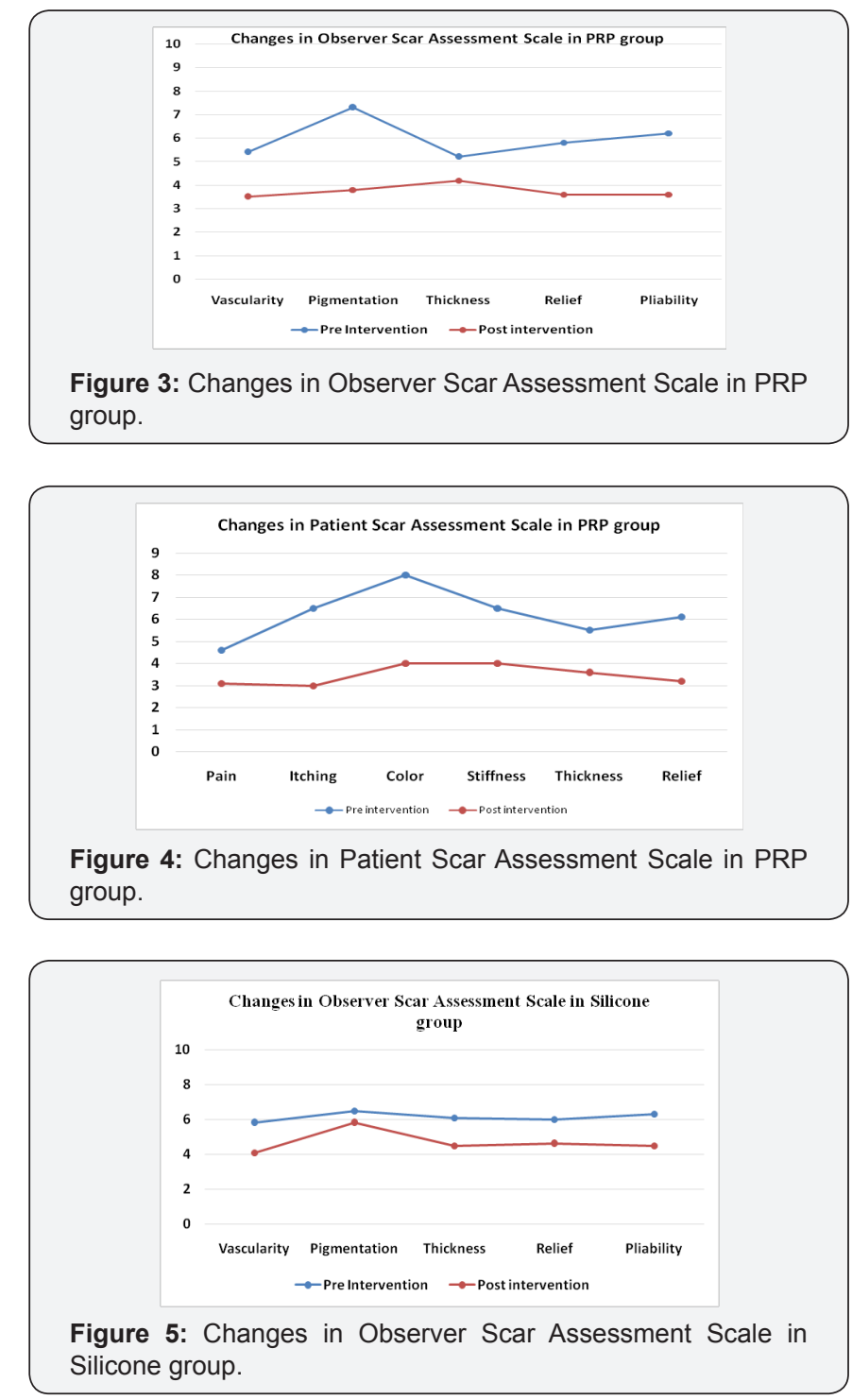

The results were evaluated one month after the last session by comparing the pre-and post-improvement photographs taken with the digital camera and Patient and Observer Scar Assessment Scale (POSAS) [10]. The results of descriptive statistics are diagrammatically shown in Figures 3-8. In Figure 3 the improvement of the parameters of the of the observer assessment among PRP group, it shows quite improvement of pigmentation, pliability and relief with mean difference $3.5,2.6$ 
and 2.2 respectively, the least improvement of these parameter was thickness with 1.0 mean difference. All results were statistically significant. Figure 4 shows the improvement of the parameters of the of the patients assessment among PRP group, it shows quite improvement of color, itching and relief with mean difference $4.1,3.5$ and 2.8 respectively, the least improvement of these parameter were pain and thickness with 1.6 and 1.9 mean difference respectively. All results were statistically significant.
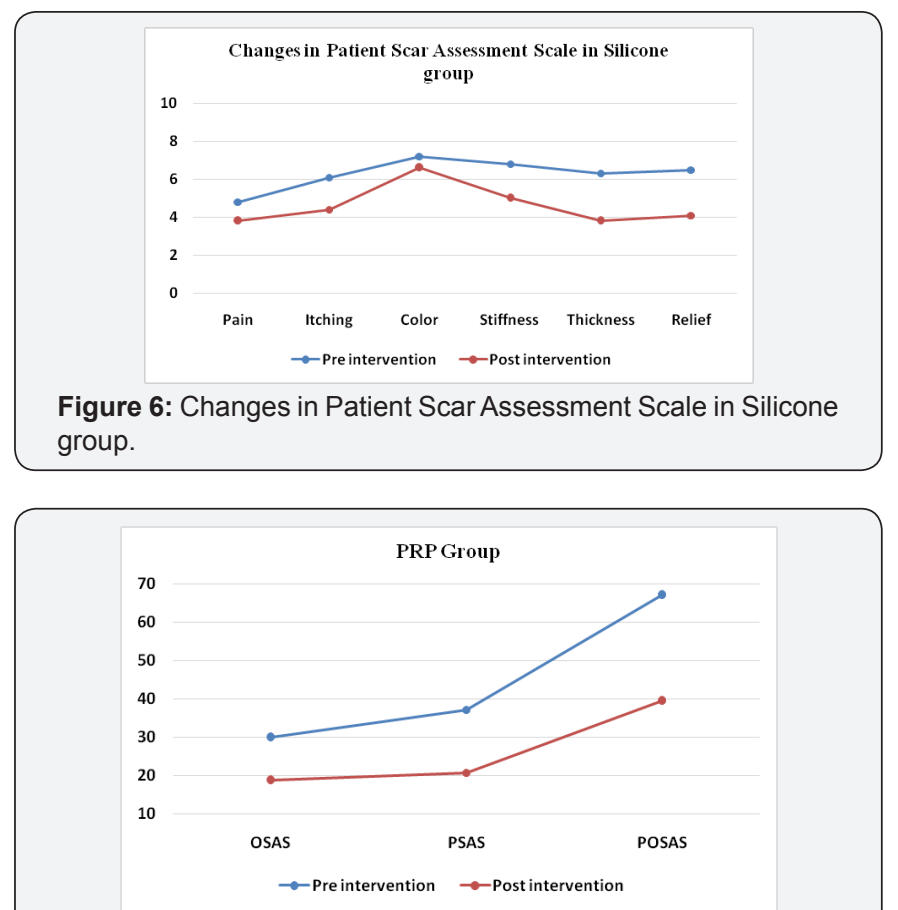

Figure 7: Changes in OSAS, PSAS and POSAS in PRP group.

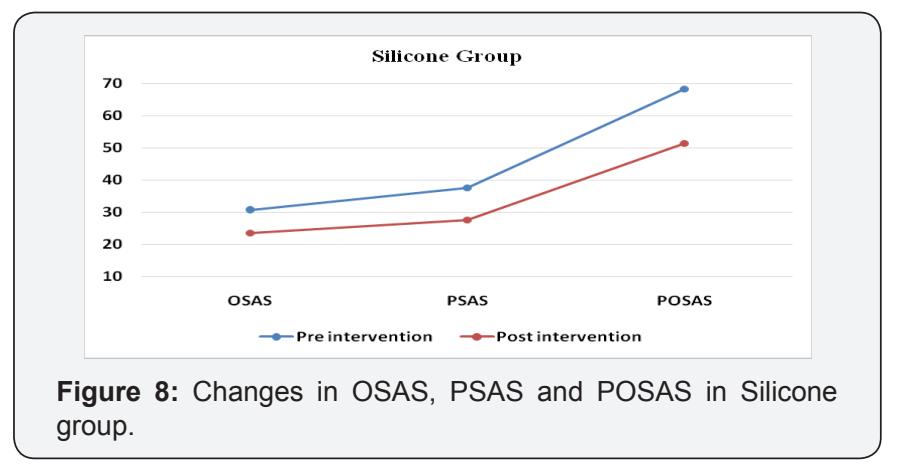

Regarding the Siliconegroup, Figure 5 shows theimprovement of the parameters of the of the observer assessment, it shows mild improvement of pliability and Vascularity with mean difference 1.8 and 1.7 respectively, the least improvement of these parameter was pigmentation with 0.7 mean difference. All results were statistically significant. While Figure 6 shows the improvement of the parameters of the of the patient assessment, it shows quite improvement of thickness and relief with mean difference 2.5 and 2.4 respectively, the least improvement of these parameter was color with 0.6 mean difference. All results were statistically significant. Figures 7 and 8 show the improvement of OSAS, PSAS and POSAS among both groups; the PRP and Silicone groups.

\section{Cases}

\section{Case 1}

a. Brief History: Four-year old female child with post scald burn scar of left arm and elbow 2 years duration (Figures $9 \&$ 10).
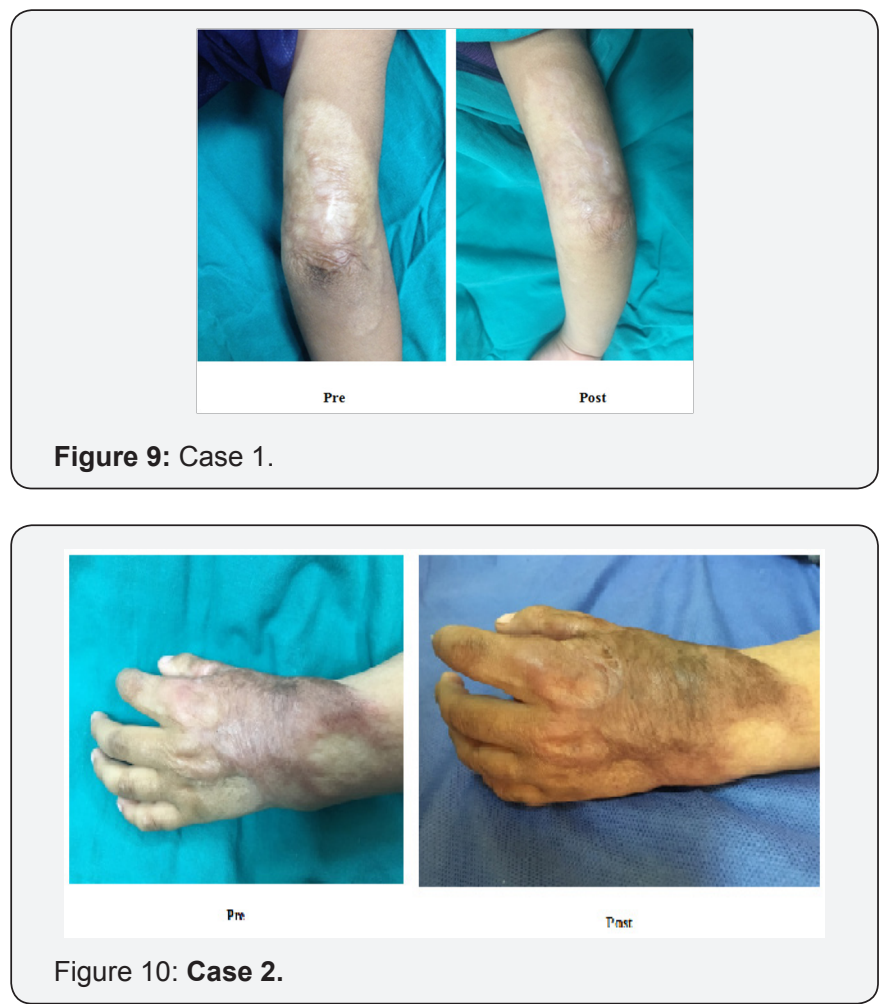

b. Procedure: 3 sessions of intralesional PRP injection with one month interval.

\section{Discussion}

Many treatment modalities have been advocated for the treatment of post burn scars but there is no universally accepted treatment protocol. Laser, surgery, radiotherapy, Silicone gel sheet, cryotherapy, interferon, 5-fluorouracil, and intralesional corticosteroids have all been used alone or in combinations, with variable success [9]. The literature has reported conflicting results on the benefits of PRP. The number of participants in different studies is usually small and the techniques used are not standardized [11]. Different platelet concentrations are achieved by means of different methodologies with results which are sometimes not well defined as to the improvement of cicatrization [12]. The increase in the rotation force is known to provide a higher platelet concentration; however, too high forces may lead to the loss of growth factors in the supernatant plasma due to an early activation of the platelets and to the rupture of the tubes which mean losses to therapeutic efficiency of PRP [13]. 
In general, the unsatisfactory results reported may be associated with the quality of the material obtained, since the platelet concentrations obtained are highly divergent among the studies. Other important variables are related to the maintenance of platelet integrity and effective activation of the material, which often are not elucidated in the studies. Platelet mean concentration of the PRP samples were 4-fold the mean concentration of whole blood samples. This result are in accordance with [14] where, an increase of up to 3-5 times than normally found in PRP samples. The study done by Phillips et al. reported a significant reduction in itching $(\mathrm{p}<0.03)$ and a decrease in pain $(p<0.08)$ after Silicone sheet treatment [15]. They attributed that to the efficacy of hydration for scar. Also Beranek [16] proposed that hydration of the scar decreases capillary activity and thus local collagen deposition.

According to this study, treatment of patients with post burn mature scar by intralesional PRP injection was found to be better than treatment of the patients by Silicone-based products. Especially in itching, pigmentation and pliability, on the other hand treatment with Silicone-based products showed better results than treating with PRP in improving the thickness of the scar. We are aware of limitations of this study, namely small number of patients, lack of experiences with applications of platelet concentrate in scars and still insufficient number of publications dealing with the scarring processes after PRP application.

\section{Conclusion}

According to the study results, treatment of patients with post burn mature scar by intralesional PRP injection was found to be better than treatment of the patients by Silicone-based products. Especially in itching, pigmentation and pliability, on the other hand treatment with Silicone-based products showed better results than treating with PRP in improving the thickness of the scar. This novel treatment appeared to be safe and effective for scar treatment. Further studies are needed to explore the potential use of this treatment.

\section{References}

1. Schultz GS, Sibbald RG, Falanga V, Ayello E A, Dowsett C, et al. (2003) Wound bed preparation: A systematic approach to wound management. Wound Repair Regen 11(1):S1-S28.
2. Foster TE, Puskas BL, Mandelbaum BR, Gerhardt MB, Rodeo SA (2009) Platelet-rich plasma: From basic science to clinical applications. Am J Sports Med 37: 2259-2272.

3. Bennett NT, Schultz GS (1993) Growth factors and wound healing: Biochemical properties of growth factors and their receptors. Am J Surg 165: 728-737.

4. Cohen S, Levi-Montalcini R (1956) A nerve growth-stimulating factor isolated from snake venom. Proc Nat Acad Sci USA 42: 571-574.

5. Raines EW, Ross R (1982) Platelet-derived growth factor. I. High yield purification and evidence for multiple forms. J Biol Chem 257: 51545160.

6. Ferrari M, Zia S, Valbonesi M, Henriquet F, Venere G, et al. (1987) A new technique for hemodilution, preparation of autologous plateletrich plasma and intraoperative blood salvage in cardiac surgery. Int J Artif Organs 10: 47-50.

7. Galliera E, Corsi MM, Banfi G (2012) Platelet rich plasma therapy: Inflammatory molecules involved in tissue healing. J Biol Regul Homeost Agents 26: 35S-42S.

8. Cervelli V, Gentile P, Scioli MG, Grimaldi M, Casciani CU, et al. (2009) Application of platelet-rich plasma in plastic surgery: clinical and in vitro evaluation. Tissue Eng Part C Methods 15(4): 625-634.

9. Gold MH, McGuire M, Mustoe TA, Pusic A, Sachdev M, et al. (2014) Updated international clinical recommendations on scar management: part 2- algorithms for scar prevention and treatment. Dermatologic Surgery 40(8): 825-831.

10. Fearmonti R, Bond J, Erdmann D, Levinson H (2010) A Review of Scar Scales and Scar Measuring Devices. Eplasty 10.

11. Longo UG, Loppini M, Berton A, Spiezia F, Maffulli N, et al. (2012) Tissue engineered strategies for skeletal muscle injury. Stem Cell Int 2012:175038.

12. Schepull T, Kvist J, Norrman H, Trinks M, Berlin G, et al. (2011) Autologous platelets have no effect on the healing of human Achilles tendon ruptures a randomized single-blind study. Am J Sports Med 39: 38-47.

13. Vendramin FS, Franco D, Franco TR (2009) Methods to obtain autologous platelet-rich plasma gel. Ver Bras Cir Plast 24: 212-218.

14. Pietrzak WS, Eppley BL (2005) Platelet rich plasma: biology and new technology. J Craniofac Surg 16: 1043-1054.

15. Phillips TJ, Gerstein AD, Lordan V (1996) A randomized controlled trial of hydrocolloid dressing in the treatment of hypertrophic scars and keloids. Dermatol Surg 22(9): 775-778.

16. Beranek JT (1990) Why dose topical silicone gel improves hypertrophic scars? A hypothesis. Surgery 108: 122.

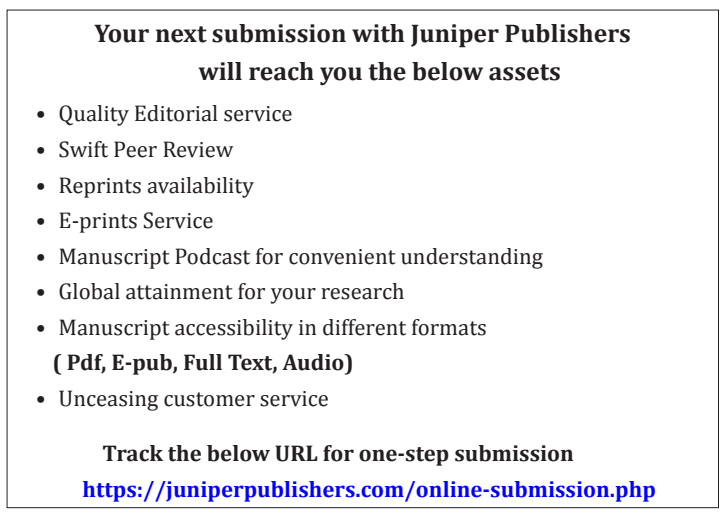

